\title{
AMÉRICA DEL SUR: DE LA SEGURIDAD COOPERATIVA A LA COOPERACIÓN DISUASORIA
}

MARina Vitelli

\section{INTRODUCCIÓN}

Suele SeÑalarse Que América del Sur constituye una región que carece de los altos niveles de rivalidad geopolítica que sufren otras latitudes, así como tampoco representa un espacio donde el extremismo -político o religioso- tenga una manifestación descontrolada, ni constituye un riesgo en materia de proliferación de armas de destrucción masiva. ${ }^{1}$ Pero si bien estas carencias alejarían a la región del conjunto de países que representan amenazas a la seguridad internacional, ellas no alcanzan para constituirla como una región completamente pacífica y armoniosa.

De hecho, la violencia en sus distintas formas -interestatal e intraestatal- forma parte de la realidad de América del Sur, tal como atestiguan las recurrentes tensiones diplomáticas entre los países de la región andina y el persistente conflicto colombiano. Las repercusiones de la Operación Fénix en marzo de 2008, a partir de la cual las fuerzas armadas colombianas atacaron un campamento de las Fuerzas Armadas Revolucionarias de Colombia (FARC) en territorio ecuatoriano, así como de la crisis de desconfianza generada por el acuerdo militar entre el gobierno colombiano y Estados

${ }^{1}$ Jorge Battaglino, "Palabras mortales. ¿Rearme y carrera armamentista en América del Sur?”, Nueva Sociedad, núm. 215, 2008, pp. 23-34; Carlos Escudé, "El experimento del bicentenario: las políticas pacifistas de la Argentina, 2003-2011", Revista Argentina de Ciencia Política, núms. 13/14, 2010-2011, pp. 13-25. 
Unidos de julio de 2009, constituyen dos de las instancias más agudas en las cuales se manifestaron las diferencias políticas existentes.

De similar manera, la retórica nacionalista que rodea las negociaciones diplomáticas de los conflictos de límites pendientes de resolución entre Chile, Bolivia y Perú, o entre Venezuela y Guyana, y más aún, las consecuencias de los mismos para las respectivas doctrinas militares, representan otros indicadores que relativizan los enfoques que acentúan los elementos cooperativos y pacíficos de la región. Estos últimos, por su lado, ponderan de forma positiva la existencia de instancias como la Unión Suramericana de Naciones (Unasur) y la Comunidad de Estados Latinoamericanos y Caribeños (CELAC), en tanto indicadores de crecientes convergencias en torno a la necesidad del diálogo político y la cooperación, aun cuando los proyectos de integración económica regional tuvieron resultados decepcionantes en relación a las expectativas iniciales.

¿Cómo entender la existencia del Consejo de Defensa Sudamericano (CDS) en el marco de esta convivencia contradictoria entre la violencia y la cooperación? ¿Cómo se compatibilizan la persistencia de desconfianzas, diferencias ideológicas y altos niveles de violencia paraestatal con la creación de una instancia de consulta, cooperación y coordinación en materia de defensa, que según su estatuto tiene entre sus objetivos "consolidar Sudamérica como una zona de paz; construir una identidad sudamericana en materia de defensa; y generar consensos para fortalecer la cooperación regional en materia de defensa"?

Algunos entienden que la pregunta sería improcedente puesto que el cDs carecería de sustancia, en virtud de las múltiples diferencias que existen entre sus miembros, tanto ideológicas como en relación a los mismos conceptos sobre seguridad y defensa ${ }^{2}$ o las limitaciones autoimpuestas por el Consejo en

${ }^{2}$ Emilse Calderón, "Brasil en el Consejo de Defensa Sudamericano: obstáculos a la cooperación y el liderazgo”, Relaciones Internacionales (GERI-UAM), núm. 18, 2011, pp. 89-111; Víctor Mijares, "Consejo de Defensa Suramericano. Obstáculos para una alianza operativa”, Politeia, vol. 34, núm. 46, 2011, pp. 1-46; José Manuel Ugarte, "El Consejo de Defensa Sudamericano: balances y perspectivas", Fundación Friedrich Ebert, 2010. 
términos operacionales y de equipamiento. ${ }^{3}$ Desde nuestro punto de vista, por el contrario, entendemos que seis años de actividad regular merecen un análisis más profundo sobre el significado del CDS dentro de la dimensión de la seguridad y la defensa regionales.

Más concretamente, sostenemos que dicho análisis debe tener en cuenta el devenir reciente del tratamiento regional de la seguridad y la defensa desde el fin de la Guerra Fría. De igual importancia es la consideración de los procesos de normalización de vínculos y pacificación de relaciones de rivalidad durante los años ochenta y noventa en el Cono Sur, en conformidad con el modelo de seguridad regional basado en la confianza y la transparencia. Mientras que estos dos factores han sido discutidos en la bibliografía existente, en el marco de este trabajo agregamos un elemento de análisis adicional. Nos referimos al hecho de que estas dinámicas de diálogo y cooperación surgidas a finales del siglo xx adquirieron una nueva orientación durante la primera década del siglo XxI en el marco de las tendencias del regionalismo postliberal, caracterizado por la primacía de la agenda política, vinculada a proyectos de liderazgo regional, la revalorización del principio de soberanía, un mayor rol del Estado ${ }^{4}$ y una relativa distancia respecto a Estados Unidos. ${ }^{5}$

A partir de esos elementos argumentamos que el modelo de seguridad regional que caracteriza al CDS constituye una metamorfosis del esquema de seguridad cooperativa construido durante los años noventa. En particular, a partir de la creación del cDs, posterior a la derrota diplomática del concepto de seguridad multidimensional en el subcontinente, América del Sur parece estar construyendo un modelo propio que ha sido definido como de cooperación disuasoria. Este modelo recupera las lecciones aprendidas a partir del anterior esquema influenciado por las tendencias

${ }^{3}$ Juan Battaleme, "Más incertidumbres que avances", DEF Online, 13 de octubre de 2010, http://www.defonline.com.ar/?p=574.

${ }^{4}$ José Antonio Sanahuja, "Del regionalismo abierto al regionalismo post-liberal. Crisis y cambio en la integración regional en América Latina”, Anuario de la integración regional de América Latina y el gran caribe, núm. 7, 2009. pp. 12-54.

${ }^{5}$ Andrés Serbin, "Regionalismo y soberanía nacional en América Latina: los nuevos desafíos", Documentos CrIEs, Buenos Aires, CRIES, 2010. 
de la inmediata post-Guerra Fría y una cierta ideología liberal, a las cuales el CDS suma elementos de tipo autonómico.

Aun reconociendo la habitual debilidad institucional de los organismos de integración y cooperación latinoamericanos, la complejidad intrínseca de la defensa nacional como área de cooperación interestatal y la relativa corta edad del CDS, entendemos que resulta posible, e incluso necesario, arriesgar reflexiones sobre el mismo. En función de esto, nuestro análisis sobre el concepto estratégico que está construyendo este organismo se basa en señalar la existencia de cuatro elementos: el modelo de la seguridad cooperativa, la concepción de la defensa nacional separada de la seguridad pública, un elemento disuasorio y la preocupación por la autonomía. A continuación, desagregaremos cada uno de estos componentes, trazando su recorrido histórico y señalando las acciones realizadas por el CDs hasta el momento en cada dimensión. El artículo concluye señalando algunas tensiones existentes en torno al concepto de la cooperación disuasoria.

\section{SEgURIDAD COOPERATIVA PARA LA CONFIANZA MUTUA}

$\mathrm{Al}$ analizar tanto el momento del surgimiento del CDS, como su misión y objetivos, resulta sencillo identificar el reconocimiento por parte de los gobiernos de la persistencia de situaciones asociadas a la desconfianza y la tensión, provocadoras incluso de episodios de conflicto que podrían desatar enfrentamientos armados. El entendimiento de que la Unasur precisaba un organismo que previniera -más que gestionara- el estallido de crisis de desconfianza se hizo presente en el momento mismo en que la propuesta brasileña de su constitución se hizo pública, en ocasión de la mencionada tensión regional provocada por la Operación Fénix. El 4 de marzo de 2008, durante un discurso en Campinas, el presidente Lula da Silva se refirió al hecho, al manifestar que "América del Sur no está preparada para conflictos", por lo cual "Brasil precisa proponer en el continente un Consejo de Defensa Sudamericano". ${ }^{6}$

${ }^{6}$ Luiz Inácio Lula Da Silva, "Discurso do presidente da República, Luiz Inácio 
Pocos días después, el entonces ministro de defensa brasileño Nelson Jobim retomó la propuesta formulada por el presidente al manifestar que, después de una amenaza de conflicto como la citada, "una organización como esa se tornó absolutamente necesaria como un mecanismo de prevención". ${ }^{7}$

Precisamente, la prevención de las tensiones surgidas por desconfianzas e incertidumbres entre Estados de una región constituye el eje central del modelo de seguridad cooperativa se arraigó en América Latina a fines del siglo xx. Como alternativa al tradicional modelo de la disuasión -en el que cada Estado busca su propia seguridad mediante el tradicional balance de poder-, la seguridad cooperativa diseña instrumentos destinados a prevenir conflictos antes de que estos ocurran, mediante premisas tales como la regulación de la proyección de poder más allá de las fronteras, la configuración defensiva de las fuerzas militares, la transparencia mutua y los intercambios de información y personal militar entre los Estados. ${ }^{8}$

La seguridad cooperativa tuvo su origen décadas atrás en otras latitudes. Concretamente, surgió como fruto de los debates ocurridos en el escenario europeo durante el periodo de distención internacional de fines de los años sesenta. Más adelante, durante la Conferencia de Helsinki, celebrada en 1975, aquellas discusiones sobre la seguridad en Europa evolucionaron para convertirse en los principios contenidos en el Acta final de la reunión. ${ }^{9}$ Aquel incremento del diálogo político entre los bloques ideológicos, destinado a incrementar la seguridad para ambos, atravesó un momento de parálisis e incluso algunos retrocesos a finales de los

Lula da Silva, na cerimônia de inauguração da Embrapa Monitoramento por Satélite”, Campinas, São Paulo, 4 de marzo de 2008. Disponible en http:/ /www.presidencia.gov.br

7 "Após crise, Jobim quer criar grupo de defesa sul-americano", Estadao, Nacional, 12 de marzo de 2008. Disponible en http:/ /www.estadao.com.br

${ }^{8}$ Juan Alberto Rial, "Hacia un sistema de seguridad cooperativa en el Cono Sur”, Relaciones Internacionales (IRI-UNLP), núm. 25, 2003, pp. 1-16.

${ }^{9}$ Emanuel Adler, "The Spread of Security Communities: Communities of Practice, Self-Restraint, an NATO's Post Cold War Transformation”, European Journal of International Relations, vol. 14, núm. 2, 2008, pp. 195-230. 
setenta. No obstante, a mediados de los ochenta el espíritu de Helsinki fue retomado en ocasión de los cambios introducidos en la política internacional con la llegada del movimiento reformista soviético liderado por Gorbachov. ${ }^{10}$

Lo que es más, aquellos estudios críticos sobre los conceptos de la seguridad en la Guerra Fría desarrollados por funcionarios y expertos de Europa del Este -sobre los cuales se basó la seguridad cooperativa- trascendieron las fronteras para ser tomados por las Naciones Unidas. Concretamente, en 1985 el Secretario General de la organización designó a un grupo de expertos para discutir sobre conceptos de seguridad y cinco años más tarde un nuevo grupo creado por la Asamblea General continuó trabajando sobre el tema hasta publicar en 1993 el documento "Estudio sobre Conceptos y Políticas de Seguridad Defensiva”.

Tal como expresa el título, la nueva perspectiva entendía que eran las actitudes y las capacidades ofensivas de los Estados las que provocaban inseguridad en la región, de manera que el dilema de seguridad basado en el conflicto ideológico estimulaba políticas de preparación para la guerra que eran aún más responsables de las desconfianzas que las mismas diferencias políticas. Era necesario, por lo tanto, transformar las posturas militares ofensivas -y las capacidades militares que las respaldaban- en defensivas para disminuir las probabilidades de un enfrentamiento.

En otras palabras, la coexistencia pacífica no bastaba; hacía falta cambiar la lógica hacia una seguridad defensiva, un modelo construido sobre la idea de la seguridad común. Esta se hacía posible si los Estados emitían un conjunto de señales políticas y militares de transparencia y confianza, porque la paz y la cooperación entre los Estados era la mejor garantía de la seguridad mutua. La lista de medidas a ser tomadas incluía la resolución diplomática de

10 Thomas Risse-Kappen, "Ideas Do Not Float Freely: Transnational Coalitions, Domestic Structures, and the End of the Cold War", International Relations Theory and the End of the Cold War, Nueva York Columbia University Press, 1995, pp. 187-222; Robert Herman, "Identity, Norms, and National Security: The Soviet Foreign Policy Revolution and the End of the Cold War", The Culture of National Security: Norms and Identity in World Politics, Columbia University Press, 1996. 
conflictos pendientes, la transparencia en la información estratégica y el control político sobre las fuerzas armadas. ${ }^{11}$

Ciertamente el hecho de que América Latina hubiera adoptado la seguridad cooperativa algunos años más tarde respondió en parte a que tal modelo resultaba funcional a la agenda hemisférica que Estados Unidos intentaba establecer, basada en la orientación general que su política exterior adquiría en los primeros momentos de la post-Guerra Fría. Puntualmente, en el contexto del proclamado Nuevo Orden Mundial que parecía surgir, este país auspiciaba la resolución de conflictos domésticos e interestatales que hasta hacía poco había alentado, a la vez que exhortaba a la desmovilización y la reforma de aquellas fuerzas armadas que pocos años antes había entrenado y equipado. La seguridad cooperativa, al proponer la resolución pacífica de los conflictos pendientes y la institucionalización de medidas de transparencia y confianza con fuerzas armadas menos ofensivas, aparecía como la nueva orientación recomendada para las políticas de seguridad regional de los países de la región.

En particular, el concepto formó parte de la perspectiva que diseñaron los funcionarios que llegaron al Departamento de Defensa con el presidente estadounidense Bill Clinton. En 1993, Ashton Carter, William Perry y John D. Steinbrunner publicaron el influyente documento titulado A New Concept of Cooperative Security, en el cual sostenían que el propósito central de la seguridad cooperativa era:

evitar la guerra a partir de impedir que los medios para una agresión exitosa sean obtenidos, obviando también la necesidad de que los Estados amenazados se preparen para contrarrestar esa acción. Así, la seguridad cooperativa desplaza el centro del planeamiento de seguridad desde la preparación para contrarrestar amenazas hacia la prevención de que estas surjan, desde la disuasión de las agresiones hacia hacer que la preparación para la agresión sea más difícil. ${ }^{12}$

${ }^{11}$ ONU, "Estudio sobre conceptos y políticas de seguridad defensiva”, Naciones Unidas, 1993.

12 Ashton Carter, William Perry y John D. Steinbrunner, "A New Concept of Cooperative Security”, Brookings Institution Press, 1993, p. 7. 
A estos dos factores de origen externo -los desarrollos en Europa y en Estados Unidos- se sumaron condicionantes de origen doméstico que volvían atractivo al modelo de la seguridad cooperativa. En América Latina, la doctrina de la seguridad nacional se había instalado sobre un histórico de rivalidades como las que existían entre Argentina, Brasil y Chile, así como conflictos limítrofes no resueltos que ensombrecían las relaciones entre algunos países andinos. Si bien estos diferendos no impidieron que los militares de algunos países colaboraran en las operaciones de represión política tales como el Plan Cóndor, tampoco desaparecieron al término del enfrentamiento ideológico entre Occidente y el mundo comunista. En ocasiones, la supervivencia de estas rivalidades con visos militares ponía en peligro a los regímenes democráticos en distintas etapas de surgimiento o consolidación, puesto que servían para continuar legitimando los roles políticos y sociales de carácter tutelar de las fuerzas armadas. Junto a otros motivos -como la adopción de orientaciones de política exterior enfocadas a la inserción internacional en un orden liberal-, la imperiosa necesidad de consolidar el poder civil llevó a algunos países a buscar soluciones diplomáticas para las controversias pendientes y a impulsar la adopción de posturas estratégicas defensivas y cooperativas con respecto a los vecinos.

Entre estos últimos, sin dudas, Argentina fue uno de los casos paradigmáticos. Este país llevó adelante conocidos procesos de pacificación de las relaciones con sus dos rivales fronterizos. Comenzando con el referéndum sobre el Canal de Beagle y la inmediata firma del Tratado de Paz y Amistad con Chile en noviembre de 1984 y luego con la resolución diplomática de los conflictos fronterizos pendientes con este país, Argentina buscó un diálogo político directo para poner fin a una de las principales fuentes de hipótesis de conflicto. En el caso de Brasil, los acuerdos para el uso pacífico de la energía nuclear -un claro ejemplo de intercambio de información estratégica- apuntaron a construir transparencia y confianza que luego cimentaron una cooperación bilateral para el desarrollo pacífico de tecnologías propias. ${ }^{13}$

${ }^{13}$ En noviembre de 1985, el presidente argentino Raúl Alfonsín y el brasileño 
De similar manera, la adhesión o ratificación de acuerdos internacionales de restricción de armamentos de destrucción masiva, así como la creación de nuevos instrumentos, constituyeron otro pilar de la adopción del modelo de seguridad cooperativa en América Latina. Así, a la ratificación del Tratado del Tlatelolco y la firma del Tratado de No Proliferación Nuclear por parte de los Estados que aún no lo había hecho, se sumaron el Compromiso de Mendoza, de septiembre de 1991, y la Declaración de Cartagena, de diciembre de 1991, por medio de los cuales el Mercosur y la Comunidad Andina, respectivamente, prohibían toda actividad relacionada con las armas químicas. Por su parte, la Declaración de Zona de Paz del Mercosur y Estados asociados (1999), de la Comunidad Andina (2002) y la Sudamericana (2002), manifestaban la renuncia de los respectivos países a toda acción vinculada a las armas nucleares.

Finalmente, otro elemento fundamental de la seguridad cooperativa fue construido alrededor de la creación de medidas de confianza mutua. A lo largo de la década de 1990 y en los años que siguieron, los países de la región fueron estableciendo -en especial de manera bilateral- un conjunto de acciones destinadas al intercambio de información militar, como presupuestos, inventarios, ubicación de unidades y comando y notificación de pruebas y maniobras; medidas de observación e inspección, así como medidas de restricción de determinadas actividades militares con el objeto de construir confianza y transparencia. ${ }^{14}$

El tema también fue objeto de análisis durante las Conferencias de Ministros de Defensa de las Américas realizadas en Williamsburg

José Sarney firmaron la Declaración Conjunta sobre Política Nuclear por la cual manifestaron los propósitos pacíficos de sus respectivos programas nucleares. En 1991 suscribieron el Acuerdo para el Uso Exclusivamente Pacífico de la Energía Nuclear que creó la Agencia Brasileño-Argentina de Contabilidad y Control de Materiales Nucleares (ABACC) y pocos meses más tarde firmaron el acuerdo cuatripartito con la ABACC y el Organismo Internacional de Energía Atómica (oIEA) para la aplicación de salvaguardias totales en todas sus instalaciones nucleares. Juan Pablo Milanese, “Argentina y Brasil: hacia un proceso de seguridad cooperativa”, Agenda Internacional, vol. 1, núm. 4, 2005, pp. 142-157.

${ }^{14}$ Isaac Caro, "Medidas de confianza mutua en Sudamérica", Estudios Internacionales, vol. 28, núm. 109, 1995, pp. 37-57. 
(1995) y Bariloche (1996), aunque el tratamiento de la seguridad cooperativa había comenzado algunos años antes de la celebración de dichas reuniones ministeriales. En el marco de los intentos por revitalizar a la Organización de Estados Americanos (oEA) a comienzos de los noventa, los países miembros llevaron el concepto al organismo cuando en 1992 se dispuso la creación de la Comisión Especial sobre Seguridad Hemisférica. En un documento titulado "Aportes a un nuevo concepto de seguridad hemisférica- seguridad cooperativa" se pasaba revista a los conceptos de seguridad, defensa y seguridad colectiva; el órgano se manifestaba a favor del remplazo del modelo clásico de alianza militar ofensivo-defensiva por el modelo de la seguridad cooperativa y se destacaba el componente preventivo de las desconfianzas y disputas que pudieran surgir alrededor de percepciones erróneas sobre las capacidades militares de los vecinos. ${ }^{15}$

Tres años después, al término de la primera reunión ministerial arriba mencionada, los Estados resumían el modelo de la seguridad cooperativa en los "Principios de Williamsburg": la preservación de la democracia en tanto base para garantizar la seguridad mutua; el reconocimiento del rol de las fuerzas armadas y de seguridad en la defensa de los legítimos intereses de los Estados democráticos; el compromiso con la subordinación de los militares al poder político democráticamente electo y el encuadramiento de sus acciones dentro de las normas constitucionales y los derechos humanos; la valoración de la transparencia en los asuntos militares a partir de los intercambios de información; la publicación de los gastos de defensa y un mayor diálogo civil-militar; la meta de la resolución pacífica de las disputas y la adopción de medidas de fomento de la confianza, al ritmo de la integración económica hemisférica con base en la interdependencia que existe entre seguridad económica y seguridad militar; y la promoción de la cooperación en defensa en lo que hace a la participación voluntaria en operaciones de paz de Naciones Unidas. El último principio de la lista -la cooperación con un rol de apoyo en la lucha contra el narcoterrorismo-

${ }^{15}$ Hernán Patiño Mayer, "Aportes a un nuevo concepto de seguridad hemisférica”, 1993. 
preanunciaba el quiebre del consenso regional respecto a las características y alcances del nuevo esquema de seguridad regional.

En resumen, hacia finales de siglo tanto las acciones implementadas por la OEA, como las iniciativas diplomáticas bilaterales en el área de la seguridad y la defensa en América del Sur, estuvieron marcadas por las premisas de la seguridad cooperativa: esto es que la manera más adecuada de garantizar la paz y la estabilidad política en la región en la post-Guerra Fría consistía en establecer medidas de prevención de los conflictos interestatales y fortalecer los sistemas democráticos, por efecto de la resolución de disputas pendientes, la renuncia al desarrollo de armas de destrucción masiva y medidas de control civil sobre las fuerzas armadas. Este modelo, al cual los diferentes Estados se plegaron con distinto énfasis, no constituye una invención autóctona, sino que encuentra sus fundamentos en el proceso europeo, los trabajos de Naciones Unidas y parte de los intereses estadounidenses. A pesar de ello, la seguridad cooperativa se transformó en un importante elemento constitutivo de la dimensión de la seguridad regional, el cual lleva a una parte de los analistas a identificar a la región con un espacio pacífico. ${ }^{16}$

Tal como hicimos referencia al comienzo del apartado, la crisis diplomática que se abrió con la Operación Fénix fue utilizada como una oportunidad para acelerar el proyecto brasileño de crear dentro de la Unasur un órgano con competencias sobre seguridad regional. Si bien los alcances no estaban del todo claros en un principio, en sus comienzos el CDS fue representado por la diplomacia brasileña como una iniciativa para prevenir la ocurrencia de nuevas crisis similares a la de marzo de 2008. Durante los meses que siguieron, en los cuales se realizaron cuatro reuniones de delegados para negociar el estatuto del CDs, otros objetivos, como la construcción de una base industrial de defensa a escala regional, se sumaron al discurso oficial brasileño. ${ }^{17}$

16 Andrew Hurrell, “An Emerging Security Community in South America?”, Security Communities, Cambridge University Press, 1998, pp. 228-264.

${ }^{17}$ Samuel Alves Soares y Raphael Camargo Lima, "No Limbo Da Dissonância: Argentina E Brasil No Campo Da Defesa", en Corival Alves do Carmo (ed.), Relações Internacionais: Olhares Cruzados, Brasilia, FUnAG, 2013, pp. 315-350. 
Así, en la redacción final del estatuto, el primer objetivo general del cDs consagra el legado de la seguridad cooperativa, al contener el elemento de prevención de conflictos y construcción de la confianza mutua: "Consolidar Suramérica como una zona de paz, base para la estabilidad democrática y el desarrollo integral de nuestros pueblos, y como contribución a la paz mundial". En efecto, las zonas de paz suponen la existencia de espacios interestatales en los cuales los miembros se comprometen a utilizar los medios de solución pacífica de controversias y de abstenerse de recurrir a la amenaza o al uso de la fuerza contra la integridad territorial de otro Estado.

Adicionalmente, el estatuto del organismo establece como objetivos específicos "fortalecer la adopción de medidas de fomento de la confianza y difundir las lecciones aprendidas" y "fomentar el intercambio en materia de formación y capacitación militar, facilitar procesos de entrenamiento entre las fuerzas armadas y promover la cooperación académica de los centros de estudios de defensa". A partir de este mandato el CDs trabajó en torno a tres cuestiones: generar transparencia en materia de gastos de defensa y capacidades militares; conformar un esquema regional de medidas de confianza mutua y construir un mecanismo de consultas frente a situaciones de tensión.

En lo que hace al primer ítem, un grupo de trabajo del cDS confeccionó una Metodología para Transparentar el Inventario Militar. Como resultado de la aplicación de la misma, en 2013 el Centro de Estudios Estratégicos del CDS (CEED) dio lugar a un Registro Suramericano de Inventarios Militares, cuya actualización será su responsabilidad. De igual manera, ambos organismos presentaron en julio de 2011 una Metodología de Medición de Gastos de Defensa, y a propuesta del equipo de trabajo que dio lugar a la Metodología se creó el Registro Suramericano de Gastos en Defensa, cuyo propósito es medir oficialmente las erogaciones que realizan los países miembros de Unasur en materia de Defensa, a partir de la definición común de gasto de defensa consensuada en la metodología, así como el resto de los estándares de presentación de la información contenidos en el documento, datos que anualmente los países miembros deben presentar ante el CEED. 
El déficit de confianza y transparencia entre los Estados de la región se volvió a manifestar con inusitada fuerza durante la tensión desatada por el acuerdo militar entre Colombia y Estados Unidos en 2009. Parte del mandato surgido de la Reunión Extraordinaria celebrada en Bariloche en agosto de 2009 para gestionar esta crisis fue diseñar un conjunto de medidas de confianza y seguridad, con que se aseguran mecanismos concretos de implementación y garantías. A estos efectos, la Presidencia Pro Témpore del cDs desarrolló, entre septiembre y diciembre de ese año, una propuesta de Procedimientos de Aplicación para las Medidas de Fomento de la Confianza y Seguridad, presentada finalmente durante el 2010.

Entre las medidas de confianza estipuladas el Procedimiento, instituyó un mecanismo de notificación de maniobras, despliegues o ejercicios militares, terrestres, aéreos o navales en las zonas fronterizas; un mecanismo de notificación de ejercicios militares con países regionales y extrarregionales; la invitación a observadores militares de los países de la Unasur a estos ejercicios y un mecanismo de comunicación entre las fuerzas militares de frontera para coordinar e informar sus actividades. Asimismo, en noviembre de 2012, los ministros del CDS encargaron al CEED la sistematización, análisis y archivo de la información recibida en relación a dichas medidas, a partir de lo cual este organismo elaboró un Mecanismo de Seguimiento de las Medidas de Fomento de la Confianza y Seguridad del CDS. ${ }^{18}$

Ahora bien, cabe destacar que la seguridad cooperativa se refiere a la dimensión externa de la defensa, entendiendo que las amenazas a la paz se dan en el nivel interestatal. Pero como señalábamos en la introducción, la inestabilidad en América del Sur viene dada principalmente por las repercusiones interestatales de la existencia

18 También vinculado al elemento de seguridad cooperativa, y como consecuencia de la crisis mencionada, a fines de 2009 Perú propuso la formulación de un Protocolo de Paz, Seguridad y Cooperación de la Unasur, a la vez que Chile presentaba el Proyecto de Decisión sobre una Arquitectura de Seguridad para Unasur, y Ecuador proponía un Proyecto de Código de Conducta sobre cuestiones de Defensa y Seguridad. Durante 2010 Perú fue la delegación encargada de los trabajos, a partir de los cuales se formuló una propuesta que tratara de manera conjunta estos proyectos que iban hacia un mismo sentido, tarea que luego pasó a la órbita del CEED. 
de amenazas provenientes de actores no estatales, es decir, del nivel de la seguridad interior o seguridad pública. Este es, sin dudas, un capítulo mucho menos consensual de la historia de la cooperación regional en la post-Guerra Fría.

\section{LA DERROTA DIPLOMÁTICA DE LA SEGURIDAD MULTIDIMENSIONAL}

Si bien la mayoría de los países coincidían en el énfasis puesto por Estados Unidos durante los noventa sobre el desarrollo de las medidas de confianza mutua, existía una discrepancia de parte de algunos Estados, fundamentalmente Argentina, Chile y Brasil, en torno a la propuesta estadounidense de involucrar a las fuerzas armadas en tareas de seguridad pública, principalmente el combate del narcotráfico, el terrorismo y otras manifestaciones del crimen organizado. Ciertamente, esta posición diplomática manifestada en los foros regionales y en las respectivas negociaciones bilaterales con Estados Unidos se contradecía con políticas domésticas tales como la utilización de fuerzas militares para las operaciones de mantenimiento de la ley y el orden en Brasil. Tanto las necesidades políticas de los gobiernos como la persistencia de sectores políticos y militares que eran favorables a la refuncionalización de las fuerzas armadas explican esta incoherencia entre lo sostenido fronteras afuera y lo que sucedía dentro del país. ${ }^{19}$

A pesar de estas ambigüedades, los países del Cono Sur resistieron las sucesivas presiones de Estados Unidos en favor de esta

${ }^{19}$ En el caso de Brasil, Martins Filho y Zirker ("The Brazilian Military under Cardoso: Overcoming the Identity Crisis", Journal of Interamerican Studies and World Affairs, vol. 42, núm. 3, 2000, pp. 143-170) sostuvieron que se daba una situación confusa en la cual esta postura -desaconsejada por los pensadores estratégicos-era adoptada de manera selectiva por el gobierno en diversas situaciones de mantenimiento del orden interno, y aceptada por un sector del Ejército como una potencial solución a la crisis de identidad militar. En lo que hace a Argentina, Saín investigó pormenorizadamente la influencia de sectores del gobierno y de las fuerzas armadas que, por motivos tanto políticos como ideológicos, intentaron que el gobierno se adecuara a la línea del combate contra el narcotráfico por medio de las fuerzas armadas (Saín, Los votos y las botas. Estudios sobre la defensa nacional y las relaciones civil-militares en la democracia argentina, Prometeo, 2010). 
modificación de las funciones militares, aun cuando estas se recrudecieron en ocasión de la puesta en marcha del Plan Colombia y más aún luego del 11-S. En el marco de los esfuerzos que en este sentido el gobierno estadounidense desplegó en la oEA, la Asamblea General del organismo realizada en 2002 en Barbados culminó con la Declaración de Bridgetown que ya esbozaba el reconocimiento de que "las amenazas, preocupaciones y otros desafíos a la seguridad en el Hemisferio son de naturaleza diversa y alcance multidimensional", tal como también sería señalado en la Declaración de la Conferencia de Ministros de Defensa de octubre del mismo año, realizada en Santiago de Chile.

En este marco de discusión regional respecto a las implicancias de las nuevas amenazas para los cambios de las fuerzas armadas latinoamericanas de post-Guerra Fría, la oEA organizó la Conferencia Especial sobre Seguridad, realizada los días 27 y 28 de octubre de 2003 en la ciudad de México, que resultó en la "Declaración sobre Seguridad en las Américas”. Este documento condensó el reconocimiento de las modificaciones en la agenda de seguridad hemisférica al sostener que:

Nuestra nueva concepción de la seguridad en el Hemisferio es de alcance multidimensional, incluye las amenazas tradicionales y las nuevas amenazas, preocupaciones y otros desafíos a la seguridad de los Estados del Hemisferio, incorpora las prioridades de cada Estado, contribuye a la consolidación de la paz, al desarrollo integral y a la justicia social, y se basa en valores democráticos, el respeto, la promoción y defensa de los derechos humanos, la solidaridad, la cooperación y el respeto a la soberanía nacional.

La adopción del concepto de seguridad multidimensional podría entenderse como una derrota de la posición de los países que coincidían en mantener un estricto concepto de seguridad estratégica basada en amenazas externas, claramente contraria a esta ampliación del concepto de seguridad. ${ }^{20}$ A pesar de esto, pocas líneas

20 Tomamos el concepto de seguridad estratégica de los trabajos conceptuales de lo que denominamos la comunidad epistémica de la defensa (Vitelli, "Ideas 
después, la declaración incluyó el reconocimiento del derecho soberano de todo Estado de:

identificar sus propias prioridades nacionales de seguridad y definir las estrategias, planes y acciones para hacer frente a las amenazas a su seguridad, conforme a su ordenamiento jurídico, y con el pleno respeto del derecho internacional y las normas y principios de la Carta de la oea y la Carta de las Naciones Unidas. [E insistía:] Los Estados del Hemisferio reconocen diferentes perspectivas sobre las amenazas y prioridades a su seguridad. La arquitectura de seguridad en nuestro Hemisferio deberá ser flexible y contemplar las particularidades de cada subregión y de cada Estado.

La inclusión de estas dos aclaraciones revela el cisma que se había producido entre los países que aceptaban la inclusión de las fuerzas armadas en tareas de seguridad interior y aquellos que habían resistido que dicha refuncionalización se tornara una premisa con amplio impacto doctrinario sobre sus fuerzas armadas, aunque lo aceptaban como medida excepcional y limitada.

A partir de esto, al promediar la década de 2000 la seguridad regional aparecía cruzada por una serie de tendencias difíciles de

y política exterior: la Comunidad Epistémica de Defensa Argentina y su rol en la cooperación regional”, Relaciones Internacionales, vol. 24, núm. 48, 2015). Estos expertos entendían que la Doctrina de la Seguridad Nacional había sido responsable de que la lógica militar se hubiera expandido hacia todos los ámbitos de la vida social: el enemigo interno mediante el cual actuaba el comunismo internacional debía ser combatido en el ámbito cultural y económico al igual que en el estratégico-militar. En el contexto de la Guerra Fría esta doctrina se había extinguido, pero la gran incertidumbre respecto a cuál sería la lógica de seguridad que la reemplazaría constituía un nuevo riesgo de ampliación del concepto de seguridad. Argumentando que la mayoría de los problemas que aquejaban a los países de la región eran desafíos de orden político-económico-social, señalaban la necesidad de dejar en claro que la utilización del instrumento militar no era adecuada ni legítima para su resolución. En virtud de esto proponían que la dimensión directamente relacionada con el uso potencial del poder militar constituyera un referente exclusivo de la noción de Seguridad Estratégica, siendo esta "un componente entre varios de un sistema total, multifacético y multidisciplinario de seguridad hemisférica". Druetta, Tibiletti y Donadío, "Los nuevos conceptos en materia de seguridad estratégica regional”, Revista SER En El 2000, núm. 1, 1992, p. 59). 
conciliar, aunque no del todo contradictorias. Por un lado, Estados Unidos impulsaba la adopción de un modelo de seguridad regional basado en tres elementos: la cooperación interestatal para el logro de la transparencia y la confianza de modo de disminuir al mínimo los conflictos interestatales entre los miembros del continente; la promoción de la participación de estos países en las operaciones de paz de Naciones Unidas; y la propuesta de que los Estados latinoamericanos disminuyeran el tamaño de sus fuerzas armadas y modificaran las funciones de las mismas hacia el combate de las nuevas amenazas, dejando -implícitamente- la defensa contra amenazas extrarregionales en manos de las fuerzas armadas de Estados Unidos. Mientras que los primeros dos elementos reunían el consenso de los Estados de la región, la división del trabajo de la seguridad regional que implicaba el último punto encontraba la oposición de países como Argentina y Brasil.

Cierto es también que esa oposición no necesariamente respondía a las mismas razones e intereses, elemento que marcó la segunda dinámica: la desactivación de los conflictos que podían detonar enfrentamientos militares en el Cono Sur, a partir del establecimiento de esquemas importantes de medidas de confianza. Pero a pesar de esos logros, el diálogo político no se había traducido en la institucionalización de la cooperación regional en defensa. Una de las razones de esta deuda pendiente era la desconfianza que despertaba en Brasil la adopción por parte de Argentina de un modelo defensivo y anclado en la seguridad cooperativa. El primer elemento significaba una renuncia a la capacidad autónoma de defensa inaceptable para los sectores soberanistas que sobrevivían a la política externa liberal de Fernando Henrique Cardoso. El segundo era visto como un modelo de seguridad regional demasiado funcional a Estados Unidos, en línea con la crítica general que se hacía de la política exterior argentina del momento y que tanta desconfianza traía al vínculo entre ambos vecinos.

A pesar de estas diferencias, y de las ambigüedades arriba señaladas, la postura oficial tanto de Brasil como de Argentina era de oposición al involucramiento de las fuerzas armadas en el combate de las nuevas amenazas como criterio de la cooperación 
regional en defensa. Esta oposición se basaba en los riesgos que implicaba para la democracia la acción de las fuerzas armadas en tareas contra un enemigo interno -preocupación fundamental entre un conjunto importante de los actores de la política de defensa argentina- el efecto de desprofesionalización de los militares como consecuencia de su participación en tareas policiales más simples y con equipamiento menos sofisticado que las tareas militares; el peligro de corrupción de las fuerzas por su exposición al crimen organizado; y, finalmente, la pérdida de autonomía estratégica que se abría al delegar en la potencia hegemónica la defensa de la región frente a amenazas externas.

Ciertamente, la fundamental divergencia entre quienes incluían la seguridad pública entre las misiones de las fuerzas armadas y quienes se oponían constituía uno de los principales dilemas a resolver para lograr la constitución del cDs. Propuesto por un país cuya posición diplomática consiste en separar la defensa externa de la seguridad interior, y respondiendo a una crisis mixta como fue la surgida por la Operación Fenix -con un componente interestatal pero con causas intraestatales-, quedaba abierta la interrogante respecto a cuál sería la concepción de la seguridad y la defensa sobre la cual se construiría el cDs.

Durante las reuniones de negociación del estatuto, las delegaciones fueron consensuando tres exclusiones. Por un lado, se decidió que el cDs constituiría un foro de diálogo y cooperación en lugar de una instancia de resolución de situaciones de amenaza o quiebre de la paz, como lo es el Consejo de Seguridad de Naciones Unidas. En segundo lugar, se descartó la inclusión de un compromiso de seguridad colectiva, excluyéndose así la posibilidad de conformación de una fuerza operativa combinada. Finalmente, se decidió que aquellas cuestiones vinculadas a la seguridad -denominadas seguridad pública, humana, ciudadana, orden público, etc.- también quedaban por fuera de la actuación del Consejo. $^{21}$

${ }^{21}$ Gonzalo García Pino, "El Consejo de Defensa Suramericano: contexto y perspectivas", en El Consejo de Defensa Suramericano de La UNASUR. Crónica de su gestación, Ministerio de Defensa de Chile, 2009, pp. 37-39. 
Ahora bien, esta exclusión no significa que los países miembros consideren que los temas de seguridad pública deban quedar por fuera de la cooperación sudamericana. De hecho, algunos meses más tarde, durante la III Reunión Ordinaria del Consejo de Jefas y Jefes de Estado y de Gobierno de la Unasur, celebrada el 10 de agosto del 2009 en Quito, los presidentes decidieron la creación del Consejo Suramericano de Lucha contra el Narcotráfico, el cual cambió su nombre a Consejo Suramericano sobre el Problema Mundial de las Drogas cuando se aprobó su estatuto en abril de 2010. De igual manera, en mayo de 2012 se celebró en Cartagena una Reunión de Ministros de Defensa, Justicia e Interior a efectos de analizar las amenazas del crimen organizado transnacional y otras nuevas amenazas a la seguridad regional. Por recomendación final del encuentro, en noviembre de 2012 fue creado en Lima el Consejo Suramericano en materia de Seguridad Ciudadana, Justicia y Coordinación de Acciones contra la Delincuencia Organizada Trasnacional.

A partir de estos datos puede observarse que al separar las problemáticas vinculadas con la seguridad interior de aquellas propias de la defensa externa, el cDs adopta el criterio político de esta separación entre las fuerzas armadas y las hipótesis de empleo que admitan un enemigo de la misma nacionalidad. ${ }^{22}$ En consecuencia, el campo de acción de la defensa queda centrado exclusivamente en la preparación para conjurar amenazas de origen externo. Ciertamente, no todos los países de la región siguen este criterio en sus políticas de defensa, razón por la cual creemos aún

${ }^{22}$ Cabe aclarar que, como señala Menezes Teixeira ("Cooperação Para a Dissuasão: o Brasil E O Conselho de Defesa Sul-Americano”, en Vitor Stuart Gabriel de Pieri y Charles Pennaforte, Defesa Nacional: Desafios E Perspectivas Geopoliticas, Rio de Janeiro, CENEGRI, 2012, pp. 173-188), puede detectarse en el estatuto del cDS cierta ambigüedad con respecto a la medida en que realmente quedan por fuera algunos temas de la agenda de la seguridad. En este sentido, en algunos de los principios nombrados en el artículo 3 se incluyen la salvaguarda del derecho internacional, de la carta de las Naciones Unidas y de la oEA, al igual que la vigencia de las instituciones democráticas y el respeto a los derechos humanos. Estos conceptos podrían dejar a discreción de los estados miembros la extensión de la esfera de la defensa hacia situaciones de carácter doméstico en lugar de agresiones externas. 
más relevante que el mismo haya sido adoptado para organizar la cooperación regional en esta materia. ${ }^{23}$

COOPERACIÓN DISUASORIA: CONFIANZA MUTUA Y BÚSQUEDA DE AUTONOMÍA

Para completar el análisis sobre el tipo de cooperación en defensa que representa el cos debe tenerse en cuenta un tercer elemento, que se suma a los estudiados en los apartados anteriores: la seguridad cooperativa y la separación entre las cuestiones de defensa externa y las de seguridad pública. La novedad que trae la cooperación disuasoria reside en que comenzaron a valorarse los mecanismos cooperativos como requisitos para que el diálogo político arribara a un conjunto de mínimos denominadores comunes de las visiones de defensa, un consenso para actuar en conjunto frente a amenazas comunes, representado en la frase usualmente utilizada por funcionarios de defensa de la región: cooperar hacia adentro para disuadir hacia afuera.

Retomando una idea arriba esbozada, cabe recordar que no estaba claro en un comienzo cuáles serían los propósitos a ser cumplidos por el CDS, de modo que se fueron agregando propuestas a medida que se negociaba su estatuto. En este contexto, uno de los indicadores que refleja la inclusión de una premisa que trasciende a aquellas de la seguridad cooperativa es el segundo objetivo general del cDs: "Construir una identidad suramericana en materia de defensa, que tome en cuenta las características subregionales y nacionales y que contribuya al fortalecimiento de la unidad de América Latina y el Caribe". Así, si bien el cDs renuncia - por el momento- a adoptar un compromiso de seguridad colectiva y a la constitución de una fuerza operacional, el trabajo orientado a formar una doctrina de defensa acorde a los intereses

${ }^{23}$ Sobre las diferencias entre las políticas de defensa de los países de la región con respecto a la participación de las fuerzas armadas en el combate de las nuevas amenazas, véase Mariano César Bartolomé, "Las fuerzas armadas sudamericanas y las perspectivas de cooperación en la lucha contra el terrorismo y el crimen organizado”, Estudios Internacionales, núm. 164, 2009, pp. 7-30. 
regionales representa la intención de diferenciarse de conceptos foráneos hacia la adopción de una visión propia. Lo que es más, este componente se asocia a la creciente convergencia en torno a la designación de los recursos naturales como un objeto de seguridad, lo cual fortalece el elemento disuasorio, aun sin designación de enemigos.

Pensar en una identidad compartida entre Estados suena "contraintuitivo" para muchos analistas de relaciones internacionales, más aún si se trata de unificar las identidades en materia de defensa nacional de países sumamente heterogéneos en términos de recursos de poder, de ambiciones políticas y niveles de desarrollo, con historias recientes de rivalidad. En otro trabajo hemos profundizado sobre las implicancias teóricas y prácticas del concepto de identidad colectiva. ${ }^{24} \mathrm{~A}$ efectos de este artículo basta señalar que la construcción de una identidad regional de defensa implica la práctica del diálogo político que permita un consenso en torno a un concepto estratégico que condense aquello que los países de América del Sur tengan en común en términos de percepciones sobre las dinámicas de inseguridad que afectan al ambiente estratégico, las amenazas y riesgos percibidos, los objetos de seguridad y los instrumentos para protegerlos. En síntesis, se trata de una faceta de la cooperación regional -la concertación política- que adquirió relevancia en América del Sur a partir de 2005, como parte de las transformaciones ocurridas en materia del tipo de regionalismo latinoamericano de los últimos tiempos, un factor relevante para comprender la actualidad de la cooperación regional en defensa en América del Sur.

De entre las interpretaciones existentes sobre los cambios y continuidades en los procesos de integración regional latinoamericanos, destacamos para el análisis del cos aquellos que contrastan el "regionalismo abierto" o "nuevo regionalismo" con el denominado "regionalismo post-liberal" ${ }^{25}$ El primero se refiere a

${ }^{24}$ Marina Vitelli, “Teoría y práctica de las identidades colectivas en las relaciones internacionales. El caso del Consejo de Defensa sudamericano”, Reflexión Politica, vol. 15, núm. 30, 2013, pp. 54-64.

${ }^{25}$ Sanahuja, "Del regionalismo abierto al regionalismo post-liberal..."; Serbin, art. cit. 
las iniciativas latinoamericanas de integración y cooperación que tuvieron lugar entre 1990 y 2005, siendo algunos de sus principales ejemplos el Sistema de Integración Centro Americano (sICA), el Pacto Andino y el Mercosur. Aun con las diferencias y deficiencias que los caracterizan, las instituciones nombradas tuvieron en común haber sido reflejos de estrategias de inserción internacional en un contexto de globalización, basadas en acuerdos centrados en la liberalización comercial, la apertura de mercados y una fuerte participación del sector privado. Ciertamente, la agenda económico-comercial resultaba clave para estos procesos: se trataba de alternativas para mejorar la inserción de las economías de la región en la globalización. Por su parte, la agenda política estuvo presente en estas iniciativas, aunque el tratamiento de los temas políticos se limitó a dos cuestiones: la defensa de la democracia y la preocupación por la solución pacífica de las controversias pendientes. ${ }^{26}$

Por el contrario, el regionalismo postliberal invierte la priorización de temas, poniendo menor énfasis en la apertura comercial para jerarquizar metas de tipo político. Este elemento de diferenciación con el regionalismo anterior se entiende cuando se analiza la naturaleza del nuevo como una respuesta a la crisis del neoliberalismo en la región y la asunción de gobiernos denominados progresistas en reacción a dicha crisis. ${ }^{27}$ La negativa a constituir el Área de Libre Comercio de las Américas (ALCA) en noviembre de 2005 y la previa coordinación de posiciones en la Conferencia de Cancún como parte de las negociaciones de la Ronda Doha de la Organización Mundial del Comercio (OMC) expresaron la estrategia de oposición a los acuerdos Sur-Norte, seguida por países como

26 Además de las declaraciones de zona de paz arriba mencionadas, dicha agenda política del "nuevo regionalismo" se reflejó en acuerdos como el Tratado Marco de Seguridad Democrática en Centroamérica de 1994, el Protocolo de Ushuaia sobre Compromiso Democrático suscripto en julio de 1998 por los miembros del Mercosur, junto a Chile y Bolivia, así como, el Protocolo Adicional al Acuerdo de Cartagena "Compromiso de la Comunidad Andina por la democracia" firmado en octubre de 1998 y la Carta Democrática Interamericana de septiembre de 2001.

27 Serbin, art. cit. 
Argentina, Brasil, Bolivia, Ecuador y Venezuela, que contrastaba con aquella de la década anterior. Esta estrategia abrió un escenario de heterogeneidad en la región, manifestada en la existencia, por un lado, de países que apuestan a modelos de inserción internacional basados en la asociación comercial con Estados Unidos y la Unión Europea (Centroamérica, Colombia, Perú y Chile con un modelo más abierto), y por otro, países que rechazaron estos acuerdos, privilegiando el control nacional sobre sus economías y la vinculación con mercados asiáticos.

Independientemente de los resultados, esta estrategia postliberal estuvo caracterizada, en palabras de Sanahuja, "por una búsqueda consciente de una mayor autonomía en el escenario internacional y en sus políticas de desarrollo, particularmente en relación a Estados Unidos". ${ }^{28}$ Sobre la exclusión de Washington coincide Serbin, agregando que en las iniciativas propias del regionalismo postliberal -como la Unasur y la CELAC- también se incluye un cuestionamiento hacia el sistema interamericano. En el ámbito de la seguridad regional, esta orientación de las políticas exteriores reforzó la oposición a la agenda estadounidense analizada en el apartado anterior, la cual se había endurecido en función de los requerimientos de la guerra contra el terrorismo emprendida. De igual manera, en países como Argentina, Brasil, Ecuador y Venezuela la valorización del rol del Estado alcanzó a la política de defensa, abriendo diversos procesos de planificación estratégica o reforma de los sistemas existentes. ${ }^{29}$ En este contexto, la Unasur,

${ }^{28}$ Sanahuja, "Regionalismo post-liberal y multilateralismo en Sudamérica: el caso de Unasur", en El regionalismo "post-liberal" en América Latina y el Caribe: nuevos actores, nuevos temas, nuevos desafíos. Anuario de la integración regional de América Latina y el gran Caribe 2012, Coordinadora Regional de Investigaciones Económicas y Sociales, 2012.

${ }^{29}$ En 2007 aprobó un Ciclo de Planeamiento de la Defensa Nacional y en 2010 actualizó su Libro Blanco. Brasil formuló su Estrategia Nacional de Defensa en 2008, introduciendo importantes modificaciones doctrinarias y aprobando proyectos de desarrollo y equipamientos de alto perfil. En el caso de Ecuador, este país dio lugar en 2011 al Plan Nacional de Seguridad Integral y a la Agenda Política de la Defensa. Venezuela, por su parte, sancionó el Plan de Desarrollo de la Fuerza Armada Nacional Bolivariana para la Defensa Integral de la Nación (Plan Sucre) 2007-2013. 
en tanto iniciativa propia del regionalismo postliberal incluyó a la defensa dentro de los temas sometidos al diálogo político, planteando como propósito construir consensos sobre enfoques y conceptos de defensa, aun reconociendo las heterogeneidades existentes en esta materia.

En este sentido, el estatuto del CDS designó como objetivo específico "avanzar gradualmente en el análisis y discusión de los elementos comunes de una visión conjunta en materia de defensa”, traducido en los planes de acción como la definición de enfoques conceptuales y la identificación de los factores de riesgo y amenazas que puedan afectar la paz regional y mundial. Este objetivo se encuentra estrechamente ligado a la creación del CEED, decidido ya en la primera reunión ordinaria del organismo, en marzo de 2009 como una instancia de producción de estudios estratégicos a efectos del asesoramiento al cDs cuando éste lo requiera. ${ }^{30}$

En cumplimiento de aquellos mandatos, el cEeD redactó el "Informe preliminar acerca de los términos de referencia para los conceptos seguridad y defensa en la región Suramericana” y comenzó a formular el "Estudio prospectivo Suramérica 2025", un análisis integral de los activos estratégicos regionales y las capacidades colectivas y su incidencia para la sostenibilidad, seguridad y defensa estratégicas de la región y su posicionamiento en el Sistema Internacional de Seguridad.

De similar manera, en el marco de la labor del CDS, entre 2010 y 2013 se realizaron tres seminarios sobre "Visiones y enfoques conceptuales de defensa, riesgos y amenazas a la región”. El último

${ }^{30}$ El CEED es el órgano dependiente del CDS que se ocupa de la realización de estudios e investigaciones en temáticas vinculadas a la defensa y la seguridad regional, a la vez que se constituye en un centro de documentación y archivo, funcional al mantenimiento de la memoria institucional del cDs. De igual manera, en lo que hace a la formación y capacitación, le cabe al CEED el establecimiento de las relaciones institucionales y la conformación de una red de intercambios entre los centros de estudios estratégicos nacionales y extra regionales. A partir de los objetivos y atribuciones mencionadas, el CEED se propone avanzar en la definición e identificación de los intereses regionales, concebidos éstos como el conjunto de los factores comunes, compatibles o complementarios del interés nacional de los países de Unasur. 
de ellos, subtitulado "Hacia una visión Suramericana para la Defensa de los Recursos Naturales y la Biodiversidad", expresaba la creciente identificación entre los riesgos y amenazas potenciales de la región y la preocupación con la protección de los recursos naturales. ${ }^{31}$ Lo que es más, el Plan de Acción de 2013 dispuso la creación de un Grupo de Trabajo para gestión y monitoreo de áreas especiales utilizando los recursos del Centro Gestor de Protección de la Amazonia en provecho de los países miembros de la Unasur, cuya labor culminó en la realización en agosto de 2013 en Manaos del I Seminario Sudamericano sobre Monitoreo de Áreas Especiales. El mismo Plan de Acción dispuso la creación de un grupo de trabajo responsable de formular un diagnóstico para proponer mecanismos de cooperación en materia de protección y defensa de los recursos naturales y la biodiversidad.

Esta vinculación entre la seguridad regional y los recursos naturales ha sido reconocida por el Director Ejecutivo del CEeD, Alfredo Forti, cuando durante su presentación en el marco del Seminario Internacional "Nuevas Dimensiones de la Integración y la Seguridad Regional”, realizado en Buenos Aires el 25 de julio de 2013, destacó el concepto de interés regional resaltando que "no hay mayor ejemplo paradigmático que ilustre este concepto [...] como los cuantiosos recursos naturales estratégicos que abundan en Suramérica y que por su diseminación, sin respetar fronteras, constituyen de hecho, activos comunes a nuestros doce países de Unasur". ${ }^{32}$ De igual manera, en la III Declaración de Lima, emitida al término de la IV Reunión Ordinaria del cDs, los ministros de Defensa convinieron en: "Reconocer la importancia de la defensa de los recursos naturales de la región, entendiendo que se debe incrementar los mecanismos de cooperación intrarregionales, así como elaborar políticas y mecanismos de disuasión ante eventuales situaciones extrarregionales que tengan implicancias y desestabilicen a Suramérica como una zona de paz".

31 Asimismo, antes del seminario mencionado, entre el 27 y el 30 de mayo de 2013, la Unasur realizó en Caracas la Conferencia sobre Recursos Naturales para el Desarrollo Integral de la Región.

32 Declaraciones disponibles en http://www.ceedcds.org.ar/Es panol/04Eventos/0016-Eventos.html 
Si bien el modelo disuasorio que parece adoptar el CDS no designa a ningún Estado o grupo de Estados como fuente potencial de amenaza hacia la región, cabe mencionar que, en ocasión del debate surgido en relación al acuerdo militar entre Colombia y Estados Unidos, y a partir de la denuncia que hiciera el presidente Hugo Chávez, la Declaración de Bariloche incluyó como mandato para el cDs el análisis del documento producido por el Comando de Movilidad Aérea estadounidense, denominado "Estrategia global de bases de apoyo". En cumplimiento de esto, durante la II Reunión Ordinaria de la Instancia Ejecutiva del CDS Venezuela presentó un informe sobre los alcances del documento, luego de lo cual se debatió sobre el tema. En una reunión posterior se decidió conformar un grupo de trabajo que revisara el texto e hiciera una síntesis objetiva del mismo, presentado en junio de 2010, conclusiones que fueron elevadas al Consejo de Jefes y Jefas de Estado y Gobierno de la Unasur.

A la preocupación sobre los recursos naturales deben agregarse dos elementos también relacionados con una voluntad de trabajar de manera conjunta en la disuasión, con mayor autonomía respecto a los modelos configurados desde Estados Unidos. En primer lugar, el cDs comenzó a atender la preocupación respecto a las amenazas cibernéticas, cuando en su Plan de Acción de 2012 preveía la conformación de un grupo de trabajo para evaluar la factibilidad de cooperación en esta área. Ciertamente, el tema cobró mayor relevancia luego del episodio de espionaje sufrido por Brasil según dio a conocer Edward Snowden en julio de 2013. Este hecho inspiró la Declaración Conjunta sobre Defensa Cibernética del 30 de agosto de 2013, luego de la Reunión del Consejo de Jefes de Estado y de Gobierno de la Unasur realizada en Paramaribo. En segundo lugar, la cooperación en materia de industrias de defensa - un objetivo autonomizante de largo plazo- es trabajado por el CDS con base en tres ejes: el trabajo de diagnóstico e intercambio de información sobre industrias militares, el proyecto de desarrollo conjunto de un Avión de Entrenamiento Básico y el diseño y producción de un Sistema de Aviones No Tripulados. ${ }^{33}$

${ }^{33}$ Con respecto a la primera cuestión, en noviembre de 2012 se realizó un 
Como consecuencia de lo expuesto hasta aquí, argumentamos que la combinación de elementos que conforman el cDs parece estar dando lugar a un modelo de cooperación en defensa original, el cual comenzó a ser designado en el discurso político y académico como un modelo de cooperación disuasoria. ${ }^{34}$ En relación al primer tipo de discurso, el ministro de defensa brasileño, Celso Amorim, ha sostenido que:

La cooperación entre nuestros países es la mejor forma de consolidar el ambiente de paz regional y, al mismo tiempo, de disuadir las amenazas externas. Tenemos así que manejarnos a través de un concepto quizás novedoso: la "cooperación disuasoria". Con objetivos como la construcción de confianza, la promoción de la transparencia, la concertación política y el desarrollo de una identidad común en defensa entre sus miembros, el CDS permitirá a los países sudamericanos trabajar permanentemente para solucionar las tensiones existentes en la región. ${ }^{35}$

En suma, el modelo de la cooperación disuasoria difiere de la seguridad cooperativa porque no se agota en la construcción de la confianza y la transparencia para evitar conflictos entre los miembros del acuerdo. A ese modelo basado en evaluaciones relativamente optimistas del entorno estratégico, la cooperación disuasoria suma la designación de vulnerabilidades estratégicas comunes y la voluntad política de atenderlas de manera conjunta. Ahora bien, la acción de disuasión no se piensa a partir de la constitución de una fuerza operacional común, ni un pacto de seguridad colectiva,

Taller para desarrollar un marco conceptual y una metodología para el diagnóstico de la industria de defensa, así como un Seminario Internacional de Tecnología Industrial Básica - Seguridad y Defensa. En materia de desarrollos conjuntos, se trabaja desde 2012 en un proyecto de construcción de un Avión de Entrenamiento Básico-Primario.

34 En el discurso académico destacamos los trabajos de Héctor Luis SaintPierre, "Brasil, el camino hacia una gran potencia: nuevo modelo de seguridad y defensa”, ATENEA Seguridad y Defensa, 2013; y Menezes Teixeira, art. cit.

35 Celso Amorim, "Discurso del Ministro de Defensa en Buenos Aires, 3 de diciembre de 2013". 
puesto que se reconoce la asimetría en términos de medios militares para enfrentarse a una efectiva agresión armada externa, así como se es consciente de que las diferencias doctrinarias entre los miembros del modelo y la persistencia de desconfianzas constituyen otros límites a esa opción. Descartada por el momento la alternativa de una disuasión a través de un pacto de seguridad, la cooperación disuasoria apuesta a la construcción de un consenso entre los miembros que genere costos políticos altos para quien esté dispuesto a amenazar los intereses regionales, una eventualidad que como subrayamos no se vislumbra en el corto ni mediano plazo.

\section{CONSIDERACIONES FINALES}

Motivados por una serie de interrogantes planteadas en la introducción, a lo largo de este trabajo abordamos mediante un recorrido conceptual e histórico aquellos factores que consideramos relevantes para comprender la actualidad de la seguridad y la defensa en América del Sur. Nuestra interpretación consistió en señalar que la pregunta respecto a cómo convive una iniciativa de cooperación en defensa como el cDs con desconfianzas, disputas ideológicas y en ocasiones incluso crisis militarizadas debía contemplar tanto la adopción del modelo de la seguridad cooperativa, como el rechazo a la refuncionalización de las fuerzas armadas y las tendencias asociadas al regionalismo postliberal. Si bien el concepto estratégico fundamentado en la cooperación disuasoria se encuentra en proceso de construcción, consideramos fundamental tener en cuenta que el CDS es reflejo de un momento político de América del Sur en el que ganaron peso las fuerzas favorables a la autonomía con respecto a las potencias tradicionales, en conjunción con aquella visión de la integración y la cooperación de tipo más horizontal.

Cabe igualmente interrogarse respecto a la medida en que esta situación irá a mantenerse o modificarse frente al agotamiento de las fuentes de poder de las cuales gozaron los países de la región durante la primera década del siglo xxI, tales como los altos precios 
de las commoditties y la apertura de espacios de participación en las instituciones de la gobernanza global. Ciertamente, los elementos de la seguridad cooperativa continuarán siendo fundamentales en el CDS, no sólo porque las desconfianzas están lejos de desaparecer, sino también en razón de las externalidades positivas que pueden generar algunas medidas de confianza mutua en términos de una futura y potencial interoperabilidad.

Por su parte, la cooperación disuasoria aún debe enfrentar una serie de desafíos para consolidarse como concepto estratégico de una identidad regional en defensa. Por un lado, la articulación de este concepto con la lucha contra el narcotráfico y el crimen organizado, actividades que aun quedando fuera del ámbito del CDS no desaparecen de las prioridades de defensa y seguridad de algunos países miembros. Asimismo, la cooperación disuasoria deberá encontrar la fórmula precisa para enfrentar el dilema de una disuasión casi imposible en virtud de la asimetría de poder militar arriba mencionada. Por último, la elección de los recursos naturales como objeto de seguridad puede despertar un rápido consenso, pero con el tiempo será cada vez más evidente que las presiones económicas y las deficiencias de los controles estatales sobre las condiciones de su explotación resultan más amenazantes que los riesgos de intervención militar foránea. Lejos de significar que la protección de los recursos naturales no debe configurar una hipótesis de empleo militar, esta última reflexión se refiere a la necesidad de pasar de un discurso político sobre estos activos hacia la identificación de los casos específicos que justificarán ser securitizados de manera regional.

Cabe preguntarse también de qué manera se verá afectado el CDs por el menor énfasis dado a la agenda postliberal que se registró en las políticas exteriores de Brasil durante el fin del primer gobierno de Dilma Rousseff y el comienzo de su nuevo periodo, así como el impacto de la Alianza del Pacífico y el devenir político incierto de Venezuela. Puede esperarse que el elemento de seguridad cooperativa perdure, en virtud de que, como dejamos asentado en el primer apartado, constituye una dimensión de la cooperación en defensa más arraigada. En comparación, el componente disuasorio del modelo en construcción probablemente 
enfrente más obstáculos para desarrollarse, aunque no deberían subestimarse las dinámicas organizacionales que están construyendo el CDS y su CEED en tanto canales de creación de conceptos y enfoques de defensa comunes.

\section{BibLIOGRAFÍA}

Adler, Emanuel, "The Spread of Security Communities: Communities of Practice, Self-Restraint, an NATO's Post Cold War Transformation”, European Journal of International Relations, vol. 14, núm. 2, 2008, pp. 195-230.

Alves Soares, Samuel y Raphael Camargo Lima, "No Limbo Da Dissonância: Argentina E Brasil No Campo Da Defesa", en Corival Alves do Carmo (ed.), Relações Internacionais: Olhares Cruzados, Brasilia, FunAG, 2013, pp. 315-350.

Amorim, Celso, "Discurso del Ministro de Defensa en Buenos Aires, 3 de diciembre de 2013".

Bartolomé, Mariano César, "Las fuerzas armadas sudamericanas y las perspectivas de cooperación en la lucha contra el terrorismo y el crimen organizado", Estudios Internacionales, núm. 164, 2009, pp. 7-30.

Battaglino, Jorge, "Palabras mortales. ¿Rearme y carrera armamentista en América del Sur?", Nueva Sociedad, núm. 215, 2008, pp. 23-34.

Battaleme, Juan, "Más incertidumbres que avances", DEF Online, 13 de octubre de 2010, http://www.defonline.com.ar/?p=574.

Calderón, Emilse, "Brasil en el Consejo de Defensa Sudamericano: obstáculos a la cooperación y el liderazgo", Relaciones Internacionales (GERI-UAM), núm. 18, 2011, pp. 89-111.

Caro, Isaac, "Medidas de confianza mutua en Sudamérica", Estudios Internacionales, vol. 28, núm. 109, 1995, pp. 37-57.

Carter, Ashton, William Perry y John D. Steinbrunner, "A New Concept of Cooperative Security”, Brookings Institution Press, 1993.

Druetta, Gustavo, Luis Tibiletti y Marcela Donadío, "Los nuevos conceptos en materia de seguridad estratégica regional", Revista SER En El 2000, núm. 1, 1992.

Escudé, Carlos, "El experimento del bicentenario: las políticas pacifistas 
de la Argentina, 2003-2011”, Revista Argentina de Ciencia Política, núms. 13/14, 2010-2011, pp. 13-25.

García Pino, Gonzalo, "El Consejo de Defensa Suramericano: contexto y perspectivas", en El Consejo de Defensa Suramericano de La Unasur. Crónica de su gestación, Ministerio de Defensa de Chile, 2009.

Herman, Robert, "Identity, Norms, and National Security: The Soviet Foreign Policy Revolution and the End of the Cold War", The Culture of National Security: Norms and Identity in World Politics, Columbia University Press, 1996.

Hurrell, Andrew, "An Emerging Security Community in South America?”, Security Communities, Cambridge University Press, 1998, pp. 228-264.

Martins Filho, João Roberto y Daniel Zirker, “The Brazilian Military under Cardoso: Overcoming the Identity Crisis", Journal of Interamerican Studies and World Affairs, vol. 42, núm. 3, 2000, pp. 143-170.

Menezes Teixeira, Augusto, "O Conselho de Defesa Sul-Americano Da Unasul: Criação Institucional E Cultura Estratégica”, en Marco Aurelio Guedes de Oliveira, Comparando a Defesa Sul-Americana, Recife, Editora Universitária UfPE, 2011, pp. 127-147.

Menezes Teixeira Junior, Augusto, "Cooperação Para a Dissuasão: o Brasil E O Conselho de Defesa Sul-Americano”, en Vitor Stuart Gabriel de Pieri y Charles Pennaforte, Defesa Nacional: Desafios E Perspectivas Geopolíticas, Rio de Janeiro, CENEGRI, 2012, pp. 173-188.

Mijares, Víctor, “Consejo de Defensa Suramericano. Obstáculos para una alianza operativa”, Politeia, vol. 34, núm. 46, 2011, pp. 1-46.

Milanese, Juan Pablo, “Argentina y Brasil: hacia un proceso de seguridad cooperativa”, Agenda Internacional, vol. 1, núm. 4, 2005, pp. 142-157.

ONU, "Estudio sobre conceptos y políticas de seguridad defensiva", Naciones Unidas, 1993.

Patiño Mayer, Hernán, “Aportes a un nuevo concepto de seguridad hemisférica”, 1993.

Rial, Juan Alberto, "Hacia un sistema de seguridad cooperativa en el Cono Sur", Relaciones Internacionales (IRI-UNLP), núm. 25, 2003, pp. 1-16.

Risse-Kappen, Thomas, "Ideas Do Not Float Freely: Transnational Coalitions, Domestic Structures, and the End of the Cold War", International Relations Theory and the End of the Cold War, Nueva York Columbia University Press, 1995, pp. 187-222. 
Saín, Marcelo, Los votos y las botas. Estudios sobre la defensa nacional y las relaciones civil-militares en la democracia argentina, Prometeo, 2010.

Saint-Pierre, Héctor Luis, "Brasil, el camino hacia una gran potencia: nuevo modelo de seguridad y defensa", ATENEA Seguridad y Defensa, 2013.

Sanahuja, José Antonio, "Del regionalismo abierto al regionalismo postliberal. Crisis y cambio en la integración regional en América Latina”, Anuario de la integración regional de América Latina y el gran Caribe, núm. 7, 2009. pp. 12-54.

, "Regionalismo post-liberal y multilateralismo en Sudamérica: el caso de Unasur", en El regionalismo "post-liberal" en América Latina y el Caribe: nuevos actores, nuevos temas, nuevos desafios. Anuario de la integración regional de América Latina y el gran Caribe 2012, Coordinadora Regional de Investigaciones Económicas y Sociales, 2012.

Serbin, Andrés, "Regionalismo y soberanía nacional en América Latina: los nuevos desafíos”, Documentos CRIEs, Buenos Aires, CRIES, 2010.

Ugarte, José Manuel, "El Consejo de Defensa Sudamericano: balances y perspectivas", Fundación Friedrich Ebert, 2010.

Vitelli, Marina, "Teoría y práctica de las identidades colectivas en las relaciones internacionales. El caso del Consejo de Defensa sudamericano", Reflexión Política, vol. 15, núm. 30, 2013, pp. 54-64.

-, "Ideas y política exterior: la Comunidad Epistémica de Defensa Argentina y su rol en la cooperación regional”, Relaciones Internacionales, vol. 24, núm. 48, 2015. 